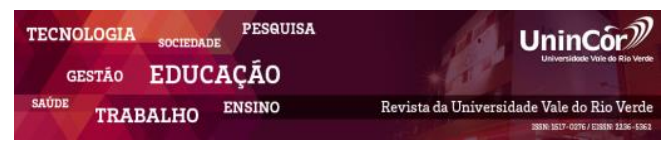

Revista da Universidade Vale do Rio Verde ISSN: 1517-0276 / EISSN: 2236-5362 v. $16\left|n^{\circ} .3\right|$ Ano 2018

\begin{abstract}
Aline Ferreira Sousa de Carvalho Universidade Vale do Rio Verde (UNINCOR) aline.carvalho@unincor.edu.br

Josenel Parreira de Castro Universidade Vale do Rio Verde (UNINCOR) josenelcastro1@gmail.com
\end{abstract}

Glei dos Anjos de Carvalho Castro Universidade Vale do Rio Verde (UNINCOR) glei.castro@unincor.edu.br

\section{RELAÇÃO DO ESCORE DE CONDIÇÃO CORPORAL SOB A INCIDÊNCIA DE RETENÇÃO DE PLACENTA E CETOSE EM BOVINOS DE LEITE}

\section{RESUMO}

A manutenção adequada do escore de condição corporal (ECC) em vacas leiteiras é importante para o controle de doenças do pósparto, incluindo a retenção de placenta e cetose. Logo, o presente trabalho objetivou determinar o efeito do ECC sobre a incidência destas enfermidades em bovinos da raça holandesa no período pós-parto. O trabalho foi realizado na Fazenda Cobiça (Três Corações, MG) utilizando-se um total de 460 vacas multíparas e primíparas que tiveram o parto entre 01 de junho de 2017 e 31 de janeiro de 2018. O ECC foi determinado em uma escala de 1 a 5 , sendo a última avaliação antes do parto considerada para caracterizar os animais. Considerou-se como retenção placentária a não liberação das membranas fetais em até 12 horas pós-parto. A cetose subclínica foi considerada quando a concentração sanguínea de $\beta$-hidroxi-butirato estava acima de $1,2 \mathrm{mmol} / \mathrm{dL}$. Ao final do período experimental obteve-se o relatório que estratifica a incidência de retenção de placenta e cetose em quatro diferentes categorias de ECC, sendo estas: ECC < 3,0; ECC entre 3,00 e 3,50; ECC entre 3,50 e 4,00 e ECC > 4,0. Os resultados demonstraram uma maior incidência de retenção de placenta nos grupos no ECC $<3$ e ECC > 4 (p < 0,05). Em relação a cetose, não houve diferença significativa entre os grupos $(p>0,05)$. Conclui-se que o ECC influência diretamente na ocorrência de retenção de placenta e visando a prevenção o ECC deve ser mantido entre 3,5 e 4,0.

Palavras-chave: Doenças pós-parto. $\beta$-hidroxi-butirato. Vacas holandesas. Membranas Fetais

\section{BODY CONDITION SCORE RELATIONSHIP IN THE INCIDENCE OF RETENTION PLACENTA AND KETOSIS IN DAIRY CATTLE}

\begin{abstract}
Proper maintenance of the body condition score (BCS) in dairy cows is of great importance for the control of postpartum diseases, including retention of placenta and ketosis. Aim with this study was to determine the effect of ECC on the incidence of these diseases in Holstein dairy cattle in the postpartum period. The study was conducted at farm Cobiça (Três Corações, MG) using a total of 460 multiparous and primiparous cows who delivered between 1 June 2017 and 31 January 2018. The BCS was determined on a scale of 1 to 5, with the last assessment before delivery considered to characterize animals. Retention of placenta was considered when there was no release of fetal membranes within 12 hours after delivery. Subclinical ketosis was considered when the blood concentration of $\beta$-hydroxy-butyrate was above
\end{abstract}


$1.2 \mathrm{mmol} / \mathrm{dL}$. At the end of the experimental period we obtained the report that stratifies the incidence of retained placenta and ketosis in four different categories of BCS: BCS < 3,0; BCS 3,003,$50 ;$ BCS 3,50-4,00 e $\mathrm{BCS}>4,0$. The results showed a significant difference between the groups for the incidence of placenta retention $(\mathrm{p}<0.05)$, the highest incidence of this disease being observed in the ECC $<3$ and ECC $>4$. For ketosis, there was no significant difference between the groups ( $p>0.05)$. It is concluded that ECC directly influences the occurrence of placental retention and, for its control, ECC should be maintained between 3.5 and 4.0 .

Keywords: Diseases; Holstein; Blood; Post childbirth; Cow.

Recebido em: 02/08/2018 - Aprovado em: 18/10/2018 - Disponibilizado em: 30/12/2018

\section{INTRODUÇÃO}

A produção mundial leiteira no ano de 2017 foi de 881 milhões de toneladas, $1,4 \%$ mais alto que em 2017. Apesar de também ter apresentado aumento na sua produção, o Brasil caiu da posição de $5^{\circ}$ para $6^{\circ}$ produtor mundial (FAO, 2018). Este setor tem grande potencial de crescimento e aumento de sua produtividade, sendo a nutrição adequada dos de grande importância para esta perspectiva, o que permite uma boa classificação de escore de condição corporal (ECC) para o animal.

O ECC é uma maneira de avaliar o armazenamento de energia dos animais e é mensurado em uma escala de 1 a 5 com intervalos de 0,25 pontos, sendo que 1 classifica o animal como magro e 5 o animal como gordo. Ambos os extremos são inadequados e afetam diretamente a saúde dos animais, resultando em desordens metabólicas, redução das taxas reprodutivas, redução na produção de leite e ocorrências de doenças pós-parto (FERNANDES et. al., 2016).

Entre as doenças do pós-parto que apresentam relação direta com o ECC pode-se destacar a retenção de placenta e a cetose. Em geral, a maior incidência de retenção de placenta é observada em vacas com baixo ou alto ECC devido à imunossupressão e maior probabilidade de desordens metabólicas, respectivamente (NOBRE et al., 2012). Porém a variabilidade na incidência da retenção de placenta pode estar relacionada a outros fatores, incluindo predisposição genética, alterações hormonais e metabólicas (BONNEVILLE-HÉBERT et al., 2011; HOSSEIN-ZADEH e ARDALAN, 2011; TONIOLLO, 2003; FERNANDES et al., 2001).

A retenção de placenta é considerada uma síndrome que pode acometer diferentes espécies animais, com maior frequência em bovinos (GAMBARINI, 2005; VASCONCELOS, 2000). A doença ocorre devido a não liberação das membranas fetais do útero por mais de 12 horas após o parto. Como consequência desta retenção, os restos placentários tornam-se meios de cultura e alimento para o crescimento de bactérias patogênicas, que podem causar infecções uterinas. Em função destas infecções, podem-se observar prejuízos tanto para a produção leiteira 
quanto para a reprodução do animal (WILTBANK, 2006).

As vacas com retenção devem ser tratadas, com antibióticos sistêmicos, ocitocina e protaglandinas, o mais rápido possível para evitar o agravamento do quadro clínico e também aumento no período de anestro (ELLIOT, 1968; PELIGRINO et al., 2008). Como prevenção é sugerido o fornecimento de mistura mineral adequada e concentrado balanceado de 20 a 30 dias antes do parto previsto. Além disso, há outras maneiras que visam contribuir para redução dos casos, como manter piquete maternidade limpo, com sombra, água a vontade, metragem de cocho compatível com o número de vacas e se possível resfriar para diminuir o estresse térmico (HAFFEZ, et. al., 2004). Adicionalmente, oferecer a devida nutrição em quantidade e qualidade suficientes para manutenção adequada do ECC, eliminar animais velhos ou com predisposição genética à retenção de placenta (FERREIRA, 1991).

Em relação à cetose, em geral a incidência é observada com maior frequência em animais com ECC superiores a 4 . Isso se deve, em parte, a maior quantidade de tecido adiposo e, consequentemente, maior mobilização de ácidos graxos das reservas energéticas para a produção de leite. Como resultado desta alta mobilização de gordura há o aumento da produção e liberação de corpos cetônicos na corrente sanguínea, principalmente acetona, acetoacetato, betahidroxibutirato (DIAS et. al., 2011; GROHN; RAJALA-SCHULTZ, 2000).

A cetose ocorrer em todas as espécies animais, assim como retenção placentária, porém acomete principalmente gado leiteiro de alta produção no início da lactação devido a necessária conversão de alimentos para produção de leite (GOFF et al., 2006; FLETCHER et al., 2001; GUARD, 2000).

A cetose pode ser classificada como clínica ou subclínica. A cetose clínica é de baixa ocorrência e subdividida em quatro tipos: primária, secundária, alimentar e espontânea, sendo descritas como: primária, o animal não recebe quantidade necessária de alimento; secundária, associada à diminuição na ingestão de alimentos, devido a outra patologia; alimentar, devido à ingestão rica em precursores cetogênicos e espontânea, quando a vaca apresenta elevadas concentrações de corpos cetônicos no sangue, mesmo ingerindo uma dieta aparentemente adequada (BARBOSA et al., 2009;:VAN CLEEF, 2009).

Os sinais clínicos da doença consistem em odor de acetona no hálito e na urina, perda de apetite, especialmente por concentrados, redução da produção leiteira e rápida perda de condição corporal. Também são observadas alterações comportamentais, sendo estas, excitação e apatia. (SOUZA, 2003; DUFFIELD, 2000).

A cetose subclínica, é de principal ocorrência, responsável pelos maiores prejuízos econômicos (BARBOSA et al., 2009). Ela é caracterizada por uma elevação dos corpos cetônicos no sangue, porém sem manifestações clínicas da doença. Na prática, animais que apresentem níveis de $\beta$-hidroxibutirato acima de 1,2 mmol/dL já são considerados com quadro de cetose subclínica (DIAS et al., 2011).

Entre os diferentes fatores que predispõe a ocorrência de cetose pode-se destacar o uso de dietas com pouca fibra e doenças que provocam 
uma diminuição na ingestão de alimentos. Além disso, sua ocorrência é maior em rebanhos de alta produção, o que resulta na queda da produtividade e na ocorrência de outros processos patológicos, como metrite e deslocamento de abomaso.

A prevenção contra a cetose é realizada por meio de manejo adequado da dieta no período seco e de transição. Como exemplo pode-se citar o uso de dieta aniônica no pré-parto e de aditivos como monensina (DUFFIELD, 2000). Esta tem função de reduzir a relação acetato: propionato, estimulando a síntese de glicose no fígado. Outro suplemento com efeito similar ao da monensina e o mais utilizado é o propilenoglicol. Ele é um precursor de glicose com a função de aumentar a ingestão de alimentos e, consequentemente, o aporte energético e diminuição da mobilização de reservas (REECE e SWENSON et al., 1996).

Diante da importância das duas enfermidades supracitadas, objetivou-se determinar a relação do ECC sobre a incidência de retenção de placenta e cetose em bovinos de leite da raça holandesa no período pós-parto.

\section{MATERIAL E MÉTODOS}

O trabalho foi realizado na Fazenda Cobiça situada em Três Corações - MG. Foram utilizadas no estudo 460 vacas multíparas e primíparas da raça Holandesa que tiveram o parto entre 01 de junho de 2017 e 31 de janeiro de 2018. Durante este período, monitorou-se o ECC, a ocorrência de retenção de placenta e cetose.
Todos os animais foram mantidos em confinamento do tipo Free Stall com cama de areia, equipado com ventiladores e aspersores de baixa pressão na linha de cocho com acesso ao piquete no pré-parto.

As avaliações de ECC foram realizadas uma vez por semana utilizando uma escala de 1 a 5 com intervalos de 0,25 , sendo a última avaliação de ECC antes do parto considerada para caracterizar os animais.

Os animais foram avaliados quanto à liberação ou não das membranas fetais até 12 horas após o parto. As fêmeas que não liberaram as membranas fetais em até 12 horas após o parto foram consideradas com retenção de placenta. No quinto dia após o parto foi coletado sangue por venopunção da veia coccígea, as coletas ocorreram entre 09:00 e 12:00 e este foi imediatamente analisado para concentração de corpos cetônicos (OptiumNeo, Xceed, AbbottLaboratories, Chicago, EUA). Os animais que apresentaram concentração de $\beta$ hidroxibutirato acima de $1,2 \mathrm{mmol} / \mathrm{dL}$ no sangue foram considerados portadores de cetose subclínica.

Os dados referentes ao ECC, retenção de placenta e cetose foram inseridos no programa de gerenciamento de rebanho (Ideagri, Belo Horizonte, Brasil). Ao final do período de avaliação dos animais os dados foram extraídos diretamente do programa em um relatório que estratificou a incidência de retenção de placenta e cetose subclínica em função do ECC. A incidência das doenças foi estratificada em quatro categorias de ECC: grupo 1: < 3,00; grupo 2: 3,00-3,50; grupo 3: 3,50-4,00; grupo 4: > 4,00. 
O teste de Qui-quadrado foi utilizado para determinar se houve diferença significativa na incidência de retenção de placenta e cetose comparando cada grupo de ECC, considerando $\mathrm{p}$ $<0,05$. Para isso, utilizou-se o programa Excel.

\section{RESULTADOS E DISCUSSÕES}

O efeito das diferentes classes de ECC sobre a incidência de retenção de placenta e cetose foi estabelecido conforme apresentado na Tabela 1. Os resultados demonstraram que houve diferença significativa entre os grupos para a incidência de retenção de placenta $(p<0,05)$, enquanto para cetose não houve diferença significativa entre os grupos $(p>0,05)$.

Tabela 1 - Relação do escore de condição corporal e a incidência de retenção de placenta e cetose em bovinos leiteiros da raça Holandesa.

\begin{tabular}{|c|c|c|}
\hline ECC & $\begin{array}{l}\text { Retenção de } \\
\text { Placenta (n/\%) }\end{array}$ & $\begin{array}{l}\text { Incidência de } \\
\text { Cetose }(\mathrm{n} / \%)\end{array}$ \\
\hline $\begin{array}{l}\text { Grupo } 1 \\
(n=31)\end{array}$ & $13 / 40,6$ & $8 / 25$ \\
\hline $\begin{array}{l}\text { Grupo 2 } \\
(\mathrm{n}=293)\end{array}$ & $47 / 16$ & $57 / 19,5$ \\
\hline $\begin{array}{l}\text { Grupo } 3 \\
(\mathrm{n}=127)\end{array}$ & $13 / 10,2$ & $16 / 12,6$ \\
\hline $\begin{array}{l}\text { Grupo } 4 \\
(\mathrm{n}=9)\end{array}$ & $3 / 33,3$ & $1 / 11,1$ \\
\hline$P$ valor & 0,0006 & 0,23 \\
\hline
\end{tabular}

A incidência das desordens pós-parto observada no presente estudo está de acordo com o relatado na literatura por outros autores (LAGO et al., 2001; CORASIN et al., 2009). No presente trabalho, foi observada uma maior incidência de retenção de placenta em animais com ECC menor que 3,00 e acima de 4,00, com menor taxa em ECC entre 3,50 e 4,00. De maneira semelhante, Nobre et al. (2012) relataram a maior ocorrência desta enfermidade para os mesmos ECC. Segundo estes mesmos autores, isto pode ser devido a ineficiência do sistema imunológico em ECC muito baixo (abaixo de 3,00) e a maior probabilidade de ocorrência de desordens metabólicas em ECC elevados (acima de 4,00).

Uma das causas para retenção placentária é a falta de manejo nutricional para cada animal de acordo com cada período de seu desenvolvimento, bem como condições locais e genéticas.

O ECC não apresentou relação com a incidência de cetose em vacas holandesas. Este resultado foi semelhante ao observado por Lago (2001). Entretanto, outros autores destacam que animais com ECC superior a 4,0 são mais susceptíveis a ocorrência de cetose, quando comparadas aqueles com ECC ideal (entre 3,00 e 4,00) (GROHN; RAJALA-SCHULTZ, 2000). Esta diferença pode estar associada a utilização de raças distintas nos estudos, assim como a temporalidade de execução dos trabalhos. Isto pode ter influência sobre as condições de manejo e nutrição destes animais e, consequentemente, possíveis alterações metabólicas.

Outro fator relacionado à ausência de relação entre o ECC e a incidência de cetose pode ser o baixo número de animais categorizados nos grupos de riscos. Talvez se fosse realizado um estudo de incidência cumulativa, durante um período mais longo e maior número de animais nestes grupos os resultados seriam diferentes. Logo, são 
necessários mais estudos para comprovar a relação deste parâmetro com a ocorrência da cetose.

\section{CONCLUSÃO}

A partir dos resultados obtidos neste trabalho, verificou-se que o ECC influencia significativamente na ocorrência de retenção de placenta em bovinos de leite. Assim, pode-se sugerir que o ECC antes do parto deve ser mantido entre 3,50 e 4,00, visando a redução da incidência desta enfermidade.

\section{REFERÊNCIAS BIBLIOGRÁFICAS}

BARBOSA, J. D., Cetose Nervosa em Bovinos, diagnosticada pela Central de diagnóstico Veterinário (CEDIVET) da Universidade federal do Pará no Período de 2000 a 2009.

BONNEVILLE-HÉBERT, A. et al. Effect of reproductive disorders and parity on repeat breeder status and culling of dairy cows in Quebec. Canadian Journal of Veterinary Research, v.75, n.2, p.147, 2011.

CORASIN, C. H., et al. Fatores de risco associados a falhas de concepção ao primeiro serviço em vacas leiteiras de alta produção. Acta Scientiarum. Animal Sciences, v. 31, n. 3, p. 311-317, 2009.

DIAS, Vagner., GARLET, Regina., ARALDI, Daniele Furian., Acetonemia em Vacas de leite. Xvi Seminário Institucional de ensino, pesquisa e extensão da Universidade de Cruz Alta. 2011. P.4.

DUFFIELD, T., Subclinical ketosis in lactating dairy cattle. Veterinary Clinics of North America: Food Animal Practice, Philadelphia, n. 16, p. 231-254, 2000 .

ELLIOT, L., McMAHON, K. J., GIER, H. T., MARIONG, G. B., Uterus of the cow After parturition: Bacterial content. Am. Journal of Veterinary Research,v.29, p.77- 81, 1968.

ORGANIZAÇÃO DAS NAÇÕES UNIDAS PARA AGRICULTURA E ALIMENTAÇÃO (FAO) Dairy Market Review, v. april, 2018, p.1-7, Rome, 2018.
FERNANDES, C.A.C. et al. Impacto da retenção de placenta sobre a performance reprodutiva de vacas leiteiras. Revista Brasileira de Reprodução Animal, v.25, p.26-30, 2001.

FERNANDES, A.F.A., OLIVEIRA, J.A., QUEIROZ, S.A., Escore de Condição Corporal em Ruminantes. Ars veterinaria, v.32, n.1, 055-066, 2016.

FERREIRA, A. M., Retenção de placenta em bovinos. Coronel Pacheco, MG. (EMBRAPA - CNPGL Documentos, 47), 1991.

FLORES R., et al. Endocrine factors and ovarian follicles are influenced by body condition and somatotropin in postpartum beef cows. Journal of Animal Science, v. 86, p.1335-1344, 2008.

GAMBARINI, M. L., et al. Infusão uterina com biguanida polimérica sobre a colonização bacteriana do útero de vacas com infecção puerperal após retenção de envoltórios fetais. Archives of Veterinary Science, v. 10, n. 2, p. 145-149, 2005.

GROHN, Y. T., RAJALA-SCHULTZ, P. J., ERB, H. N., An epidemiological and genetic study on registered diseases in Finnish Ayrshire cattle. Acta Veterinária, v. 27, n. 7, p. 182-189, 1986.

GUARD, C. L., Fresh cow problems are costly: Culling hurts the most. Hoard'sDairyman, v. 141, p.8, 1996.

GOFF, J., Principais síndromes que acometem as vacas leiteiras no período periparto.

http/:www.rehagro.com.br/siterehagro/publicaca, acesso 27/04/2017, 16:21.

HAFEZ, E. S. E., Reprodução Animal. $7^{\circ}$ Ed. São Paulo: Manole, 2004.

HOSSEIN-ZADEH, N.G.; ARDALAN, M.Cow specific risk factors for retained placenta, metritis and clinical mastitis in Holstein cows. Veterinary Research Communications, v.35, n.6, p.345-354, 2011.

LAGO, E. P., et al. Efeito da condição corporal ao parto sobre alguns parâmetros do metabolismo energético, produção de leite e incidência de doenças no pós-parto de vacas leiteiras. Revista Brasileira de Zootecnia, v. 30, n. 5, p. 1544-1549, 2001.

NOBRE, M. M., et al. Avaliação da incidência e fatores de risco da retenção de placenta em vacas mestiças leiteira. Arquivos Brasileiro de Medicina Veterinária e Zootecnia, v. 64. n.1, p. 101-107, 2012.

PELIGRINO, R.C., ANDRADE, L.R.M., CARNEIRO, L.F., Retenção de Placenta em Vacas. 
Revista Cientifica Eletrônica de Medicina Veterinária - ISSN: 1679-7353. Garça/SP. Ano VI - Número 10 janeiro de 2008 - Periódicos Semestral.

SOUZA, Alexandre N. M. Seminário apresentado na disciplina de Bioquímica do Tecido Animal do Programa de Pós-Graduação em Ciências Veterinárias da UFRGS, 2003/1.

SWENSON, J. M., REECE, W. O., Distúrbio do Metabolismo dos Carboidratos e Gordura. DUKESFisiologia dos Animais Domésticos, 11 ed, Guanabara e koogan, pp.447-448-450-452, 1996.

TONIOLLO, G.H., VICENTE, W. R. R. Manual de obstetrícia veterinária: Retenção Placentária. São Paulo, Varela. p. 104 - 105, 2003.

VAN CLEEF, H. Eric M., Sc. Distúrbios Metabólicos por Manejo Alimentar Inadequado em Ruminantes: Novos Conceitos, Rev. Colombiana cienc. Anim. 1(2). 2009.

VASCONCELOS, J. L. M. Retenção de placenta em gado leiteiro: efeito dos tratamentos hormonais com reflexos na contaminação uterina pós-parto. Milk

Point, nov. 2000.

WILTBANK, M.C., Prevenção e tratamento da retenção de placenta. In: Curso Novos Enfoques na Produção e Reprodução de Bovinos, Anais

Uberlândia, p. 61-70,2006.

\footnotetext{
Aline Ferreira Sousa de Carvalho

Médica Veterinária, Mestre e Doutora em Ciências Veterinárias pela Universidade Federal de Lavras (UFLA). Docente no curso de Medicina Veterinária da Universidade Vale do Rio Verde (UNICOR), Três Corações, Minas Gerais.
}

Josenel Parreira de Castro
Graduado em Medicina Veterinária pela
Universidade Vale do Rio Verde (UNICOR), Três
Corações, Minas Gerais.

\section{Glei dos Anjos de Carvalho Castro}

Médica Veterinária, Mestre e Doutora em Ciências Veterinárias pela Universidade Federal de Lavras (UFLA). Docente no curso de Medicina Veterinária da Universidade Vale do Rio Verde (UNICOR), Três Corações, Minas Gerais. 